\title{
Il senso del religioso nella narrativa di Tabucchi, Celati e Bufalino
}

Charles Klopp

\section{(2) OpenEdition}

Journals

Edizione digitale

URL: http://journals.openedition.org/cei/215

DOI: $10.4000 /$ cei. 215

ISSN: 2260-779X

Editore

UGA Éditions/Université Grenoble Alpes

\section{Edizione cartacea}

Data di pubblicazione: 15 juillet 2009

Paginazione: $245-253$

ISBN: 978-2-84310-145-8

ISSN: $1770-9571$

Notizia bibliografica digitale

Charles Klopp, «ll senso del religioso nella narrativa di Tabucchi, Celati e Bufalino», Cahiers d'études italiennes [Online], 9 | 2009, online dal 15 janvier 2011, consultato il 28 mars 2021. URL: http:// journals.openedition.org/cei/215 ; DOl: https://doi.org/10.4000/cei.215 


\title{
IL SENSO DEL RELIGIOSO NELLA NARRATIVA DI TABUCCHI, CELATI E BUFALINO
}

\author{
Charles Klopp \\ Ohio State University
}

Il pensiero cattolico in Italia è stato sempre una parte fondamentale della vita intellettuale del paese. Oggi, dopo un periodo di relativo disinteresse per argomenti religiosi da parte di molti pensatori di sinistra, c’è un nuovo interesse per i problemi tradizionali della religione. Molti dei commentatori su argomenti religiosi di questi giorni non si considerano cattolici o, in alcuni casi, affatto credenti - anche quando trattano argomenti come l'esistenza di Dio, la natura del peccato e l'importanza dell'incarnazione. I libri, gli articoli e le interviste di questi autori, inoltre, sono spesso indirizzati non a lettori specializzati ma al lettore generale - un pubblico di massa. Quasi tutti trattano gli argomenti in questione da punti di vista specificatamente non religiosi o religiosi soltanto in un senso culturale molto ampio (Croce).

Due degli esempi più noti di recenti pubblicazioni di questo tipo sono Credere di credere di Gianni Vattimo (Vattimo, 1996), e In cosa crede chi non crede? di Umberto Eco e Carlo Maria Martini (Eco e Martini, 1996). Nel suo breve libro, Vattimo racconta la storia del suo incontro da bambino con la fede e la Chiesa, le sue disillusioni durante l'adolescenza e il ritorno, da uomo maturo, alla riconsiderazione della possibilità di riabbracciare la fede che conosceva da bambino, seppure adesso in un modo diverso e tutt'altro che ortodosso. Il libro di Eco e Martini non contiene una confessione personale come quello di Vattimo. Ė, invece, un dialogo a volte abbastanza spiritoso da parte di due interlocutori diversi fra di loro ma allo stesso tempo rispettosissimi delle altrui posizioni. Gli avversari o interlocutori amichevoli di questo libro sono il noto semiologo, narratore e commentatore culturale - il "non credente" Eco - e l'arcivescovo di Milano e ovviamente credente Martini. Altri libri recenti o 
recentissimi che affrontano problemi di questo tipo da simili punti di vista includono Il cristianesimo di un non credente di Salvatore Natoli (Natoli, 2002), e Abramo contro Ulisse. Un itinerario alla ricerca di Dio di Filippo Gentiloni. Natoli è un filosofo e accademico, Gentiloni un giornalista che scrive spesso su argomenti religiosi.

In aggiunta alle sue moltissime pubblicazioni scientifiche, Gianni Vattimo ha dedicato un certo numero dei suoi scritti più recenti ai problemi della fede. In questi libri, il filosofo presenta le sue riflessioni su una materia di solito considerata parte del pensiero religioso - in particolare l'importanza dell'incarnazione per il pensiero cristiano. Nei suoi saggi e libri su questi e altri argomenti, Vattimo sottolinea l'importanza del declino delle grandi narrative filosofiche, per lo più derivate dall'Illuminismo, che in anni passati stavano alla base del pensiero moderno ma che al giorno d'oggi hanno perduto molto della loro forza persuasiva. In un clima intellettuale in cui le grandi narrazioni di una volta non sono più ritenute valide, la fede religiosa - o almeno la possibilità di una fede religiosa - è diventata una prospettiva più allettante di quanto non fosse dalla fine del Settecento fino a oggi. Uno dei testi in cui Vattimo spiega la sua posizione è Religione, un'opera scritta insieme a Jacques Derrida e HansGeorg Gadamer (Vattimo, Derrida e Gadamer, 1996). Un altro suo libro su argomenti simili, questa volta scritto con il filosofo Pierangelo Sequeri e il giornalista Giovanni Ruggeri, è Interrogazioni sul cristianesimo. Cosa possiamo ancora attenderci dal Vangelo? (Vattimo, 2000). Ancora più recentemente, c'è la raccolta di saggi scritti interamente da Vattimo, Dopo la cristianità: per un cristianesimo non religioso (Vattimo, Sequeri e Ruggeri, 2002). E proprio nei giorni precedenti a questo convegno ne è uscito un altro, questa volta in chiave autobiografica e scritto con Piergiorgio Paterlini: Non essere Dio (Vattimo e Paterlini, 2006).

È evidente già dai loro titoli che in questi libri Vattimo e gli altri pensatori menzionati vogliono a tutti i costi evitare l'etichetta di "cristiano" o "credente" in favore di altri termini che possano più chiaramente indicare la distanza fra le loro riflessioni e il discorso religioso convenzionale. Natoli definisce la sua fede "la cristianità di un non credente". Eco, che parla nel corso del suo libro della sua "religiosità laica" (p. 70), si domanda "che cosa crede chi - come lui - non crede". E Vattimo propone una "cristianità non religiosa”.

Nei libri di questi autori laici o non credenti o non religiosi è evidente una nuova enfasi non tanto su articoli di fede acquistati da una tradizione secolare o dalle proprie esperienze personali, quanto sulla speranza di qualche cosa che si auspica accada nel futuro. Quasi tutti gli scrittori in

$$
246
$$


questione esprimono la loro diffidenza verso sistemi totalizzanti - siano essi politici, filosofici o religiosi. Gentiloni, forse lo scrittore più politicamente militante del gruppo e abituale collaboratore al quotidiano Il Manifesto, insiste che: "Il Dio della certezza è totale, metafisico, senza incrinature" mentre "il Dio della speranza è povero, insicuro, contraddittorio come ogni speranza che, insieme, ha e non ha" (p. 32). Nel suo caso, almeno, l'agnosticismo religioso va a pari passo con un senso della necessità di stabilire un'umana solidarietà con i poveri e abbandonati, ovunque si trovino nel mondo.

La diffidenza da parte di questi scrittori per il potere e la religione istituzionalizzata deriva in parte dal loro rifiuto di sistemi totalizzanti di qualsiasi tipo, ma in parte anche da una loro valutazione spassionata delle attività della Chiesa attraverso i secoli. "Il Dio totale, onnipotente," scrive Gentiloni, "è il Dio idolo che molte pagine della Bibbia rifiutano, ma che le chiese hanno la continua tentazione di far rivivere" (p. 3I). "Un Dio totale," continua, "un Dio sicuro e la sicurezza è la maggiore forza che esista. Spesso è anche la peggiore, è la fonte di tutti i totalitarismi e di tutte le oppressioni” (p. 30).

Come Gentiloni, anche Vattimo vorrebbe distanziarsi da un passato dominato da sistemi religiosi forti. Da esponente del pensiero debole, auspica un'era di "emancipazione attraverso la consumazione delle strutture forti - del pensiero, della coscienza individuale, del potere politico, dei legami sociali, della stessa religione". Una tale liberazione, per Vattimo, fornirà "un modo di trascrivere, in termini di filosofia della storia, il messaggio cristiano dell'incarnazione di Dio, che in san Paolo si chiama anche kenosis, cioè abbassamento, umiliazione, indebolimento di Dio" (Vattimo, 2002, p. 97).

Qui e altrove nei libri di questi pensatori, il totalitarismo nella politica è considerato l'equivalente di sistemi totalitari nei campi della storiografia, della filosofia e della religione.

Per molti di questi scrittori, il rifiuto del totalitarismo e della metafisica porta a una nuova enfasi sull'etica. "Chi non crede," scrive Eco, "ritiene che nessuno lo osservi dall'alto e quindi sa anche che - proprio per questo - non c'è neppure qualcuno che possa perdonare. Questo lo sa, dall'intimo delle sue fibre, e quindi sa che dovrà in anticipo perdonare gli altri. Altrimenti come si potrebbe spiegare che il rimorso sia un sentimento avvertito anche dai non credenti?" (p. 77). Come Eco in questo passo, anche Vattimo, Gentiloni e Natoli insistono sulla necessità, in un mondo post-metafisico ma non perciò senza una dimensione etica, dell'amore e della tolleranza nei confronti del prossimo e del diverso. 
In questi testi, Dio si presenta molto spesso come una mancanza inquietante piuttosto che una presenza rassicurante. Tale mancanza, però, presuppone la possibilità di una presenza, esattamente come la nostra constatazione dei limiti dell'umano suggerisce la possibilità di un ente senza limiti. Per Natoli "non c'è infatti finitezza senza infinito [...] ma l'infinito attuale è solo di Dio, ammesso che un Dio esista" (Natoli, 2004, p. 47). Per Vattimo, in termini simili,

proprio perché il Dio-fondamento ultimo, e cioè la struttura metafisica assoluta del reale non è più sostenibile, perciò stesso è di nuovo possibile credere in Dio. [...] Se non c'è più una filosofia (storicistica, come lo hegelismo e il marxismo; o positivistica, come le varie forme di scientismo) che ritiene di poter dimostrare la non esistenza di Dio, noi siamo di nuovo liberi di ascoltare la parola della Scrittura (Vattimo, 2002, p. 9).

Oppure, nelle parole di Natoli, "se l'uomo guarda in se stesso, lui, fatto ad immagine, dovrebbe in qualche modo vedere Dio, cogliere i tratti del suo volto" (Natoli, I999, p. I7). Questo è vero anche se Dio si fa percepire all'uomo. "Nell'uomo Dio non si rende manifesto in presenza, ma è, come dire, immagine dell'assenza. Più esattamente, è l'esperienza della mancanza. Dio si imprime nell'uomo come bisogno, desiderio. Dio è lo Streben dell'uomo, è il suo spasmodico tendere." (Ibidem)

Nella narrativa italiana degli ultimi decenni c'è stata una simile svolta nell'atteggiamento degli scrittori verso temi e problemi religiosi. Già nel 1978, in una conferenza ad Amherst College nel Massachusetts, Italo Calvino aveva descritto i cambiamenti profondi nel pensiero europeo e americano durante il periodo appena concluso. Per lui si trattava di una specie di rivoluzione: "qualcosa che ha cambiato in profondità molti dei concetti con cui avevamo avuto a che fare, anche se si continua a chiamarli con gli stessi nomi" (Calvino, pp. 286-287). Tali concetti, per Calvino, includevano "quelle che sembravano essere categorie antropologiche stabili: ragione e mito, lavoro ed esistenza, maschio e femmina, e perfino le polarità delle topologie più elementari: affermazione e negazione, sopra $\mathrm{e}$ sotto, soggetto e oggetto" (ibidem). Questi cambiamenti aprivano le porte all'entrata nel mondo narrativo italiano di temi e preoccupazioni non trattati durante l'epoca del neorealismo e anni seguenti.

Anche gli storici della letteratura del periodo hanno notato questi cambiamenti di atteggiamento. Nella sua Storia letteraria del 'goo italiano, Massimiliano Capati si trova d'accordo con ciò che è stato detto da altri a proposito della non più pertinenza della tradizione illuministica e marxista per gli scrittori di oggi. Per Capati, il tramonto delle tradizioni illuministiche e razionali era il preludio di un nuovo periodo in cui l'ir-

$$
248
$$


razionale e anche il sacro avanzavano sul palcoscenico. "Oscurato," scrive Capati in termini che ricordano quelli di Vattimo, "da una lunga tradizione razionalista - illuminismo, idealismo, laicismo, marxismo - tornava a riaccendersi l'interesse per il sacro, la teologia, l'irrazionale, le superstizioni" (Capati, p. 218). Similmente, ma avvicinandosi all'argomento da una prospettiva diversa - quella della storia della stampa in Italia invece di quella della letteratura - un altro storico del mondo delle lettere italiane, Giovanni Ragone, ha anche lui identificato nella narrativa degli ultimi anni del secolo scorso un analogo, significativo "orientamento verso la realtà extra-semiotica, il comportamento, il corpo, l'inconscio” (Ragone, p. 227).

Anche se diversissimi l'uno dall'altro in stile e approcci letterari, i tre romanzieri che vorrei chiamare in causa qui - Antonio Tabucchi, Gianni Celati e Gesualdo Bufalino - fanno parte di questa svolta verso l'irrazionale, lo spirituale, il religioso. I loro scritti, sebbene in modi talmente diversi l'uno dall'altro da suggerire che qui forse succeda qualche cosa di più generale, trattano spesso argomenti che possiamo chiamare religiosi o parareligiosi.

Ecco, per esempio, cosa dice il non religioso Tabucchi a proposito degli argomenti etici che si trovano così frequentemente al centro dei suoi libri.

"Io sono un laico," spiega in un'intervista recente a Carlos Gumpert, non un cattolico. Tuttavia credo che esistano delle regole che bisogna rispettare e queste regole, in fondo, le ha inventate la religione, l'etica religiosa basata sui dieci comandamenti, che sono stati il fondamento di tutte le etiche posteriori, perfino di quelle a carattere laico. Questi dieci comandamenti mantengono ancora il loro potere di seduzione su di me. (Gumpert, pp. IOI-IO2)

Nei libri di Tabucchi queste regole sono alla base delle tante riflessioni di questo autore sulla presenza del male nel mondo, l'inevitabilità del rimorso (menzionato anche da Eco) e il senso di colpa che tutti sentono nei confronti di questo male, insieme alla necessità di trovare espiazione per aver fatto del male - se non in un contesto istituzionale, magari nel foro più laico e secolarizzato della letteratura. La disamina di coscienza, inoltre, che Tabucchi propone non si limita ai suoi personaggi o al suo io narrante, ma si estende con grande insistenza anche ai suoi lettori, nei quali l'autore vorrebbe stimolare una "inquietudine" morale che li costringa a esaminare le loro coscienze a proposito dei problemi sollevati dalle sue narrazioni. "Di una sola cosa forse posso essere orgoglioso," dice nella stessa intervista con Gumpert, "di non essere uno scrittore che placa le coscienze, perché credo che chiunque mi legga riceva per 
lo meno una piccola dose di inquietudine e chissà che un giorno questa inquietudine non finisca per germogliare e dare i propri frutti" (ibidem, p. I04). Dentro il confessionale costruito dal Tabucchi nei suoi racconti sta un lettore costretto dalle provocazioni dei testi tabucchiani a esaminare la propria coscienza e forse agire diversamente nel futuro (cfr. ibidem, p. 74).

Gli scritti di Gianni Celati possono apparire forse meno impegnati eticamente che non quelli di Tabucchi. Ma essi contengono, nondimeno, molti passi che alludono a presenze extra-umane che forse si potrebbero collegare a "l'immagine dell'assenza" e "l'esperienza della mancanza" menzionate da pensatori come Gentiloni, Vattimo e Natoli. Esempi nella produzione narrativa di Celati comprendono la storia della casalinga che decide che c'è qualche cosa al di fuori della vita che ha vissuto finora quando un meteorite cade nel suo giardino; il viaggiatore in Africa che intuisce delle presenze al di là dalla sua comprensione che sono intrinseche alle terre e alle culture che sta visitando ma che non riesce a descrivere; oppure i giovani "pascolanti" presi nei legami di un "desiderio che s'affaccia assieme ad ansia e disagio" anche se poi capiscono che "se i piaceri potessero parlare direbbero com'è deserto il mondo" (Celati, I987; 1988, p. 99; 2006, pp. 44-45).

In queste e altre allusioni a presenze misteriose nell'ambito quotidiano delle esperienze più banali, Celati sta cercando, direi, non tanto di identificare la cosa a cui si riferiscono o alludono in questi passi, quanto di specificare in quali circostanze lo "Streben" o "spasmodico tendere" descritto da Natoli possa accadere. Nelle sue riflessioni sulla desacralizzazione della vita contemporanea e la resistenza umana a un tale impoverimento spirituale - non importa se in Africa, il paese fantastico dei Gamuna, o il territorio più familiare di Ferrara e la Valpadana - Celati non auspica un ritorno ai valori religiosi tradizionali. Propone piuttosto la sua arte come un ponte verso l'ignoto, la testimonianza di una mancanza profondamente sentita da esseri umani di diverse classi sociali e provenienze nazionali - anche se quasi tutti loro (lo scrittore, forse, incluso) non siano in grado di identificare di che cosa sono stati testimoni e tanto meno identificare questo qualcosa con precisione.

Dei tre scrittori considerati qui, Gesualdo Bufalino è colui che si definisce più facilmente scrittore religioso. Negli scritti di Bufalino l'io parlante non è mai secondario come nella narrativa di Celati. Tanto meno riesce un co-investigatore tutt'altro che disinteressato e magari colpevole anche lui come negli scritti di Tabucchi. Al contrario, i narratori bufaliniani sono sempre delle presenze fortissime che manipolano il testo e $\mathrm{i}$ 
lettori per fini particolari determinati dall'autore. I lettori di Bufalino si trovano perciò forzati da narratori energici e insistenti ad affrontare direttamente e ripetutamente questioni di grande importanza quali l'esistenza di Dio e le possibilità umane di raggiungere uno stato autentico di grazia. Gli scritti di Bufalino sono altamente teatrali, perfino melodrammatici, caratterizzati da un lessico ricchissimo e pieni di metafore spesso azzardate - eccessivi, perciò, in tutti i sensi. Ma è anche evidente che una tale prosa trasgressiva e ai limiti dell'ostilità è analoga all'atteggiamento trasgressivo di questo autore nei confronti della fede religiosa. L'atteggiamento blasfemo di Bufalino è quello di un credente, ex-credente, oppure paracredente. È stato suggerito che Bufalino è ossessionato dalla "sacralità della parola" (Acunti, citato in Spunta, p. 299). Ma è anche uno che diffida di, e forse detesta, tutto ciò che è sacro: non solo la parola sacra della Bibbia e del discorso tradizionale religioso, ma anche le parole che si sente costretto a scrivere lui stesso.

Sarebbe possibile elencare altri scrittori italiani delle ultime generazioni che fanno parte di questo ritorno a un "senso del religioso" - uno sarebbe il "cannibale bianco" Giulio Mozzi, i cui scritti sono ben noti al collega e organizzatore di questo convegno Alain Sarrabayrouse - ma i lavori di Tabucchi, Celati, e Bufalino documentano sufficientemente la svolta in questa direzione dalla parte almeno di questi tre scrittori importantissimi per la narrativa degli anni '8o, '9o e oltre. In aggiunta alla posizione importante di questi argomenti religiosi in senso lato nei loro scritti, Tabucchi, Celati e Bufalino condividono anche altre posizioni artistiche e intellettuali. Una è il loro rifiuto della Storia come metanarrativa, la loro diffidenza per asserzioni che la storia proceda in una direzione specifica. Un'altra è il senso di impaccio o sconforto che sperimentano a proposito dei propri scritti, specialmente quando trattano argomenti religiosi come quelli che ho cercato di documentare. Infine, in tutti questi scrittori c'è un vivo senso dei limiti del rappresentabile e della quasi impossibilità di fare altro che alludere al vero oggetto delle loro meditazioni. I filosofi e altri scrittori menzionati all'inizio di questo intervento descrivono Dio come mancanza o assenza. Per parte loro, Tabucchi, Celati, e Bufalino dirigono l'attenzione dei loro lettori all'ineffabile o l'indicibile che sta dietro o fuori o comunque dall'altra parte dei loro racconti. Secondo Celati nel suo racconto I lettori di libri sono sempre piu falsi, la funzione delle parole nelle comunicazioni normali è di "sbarrare il passo ad un'apparenza estranea e senza facoltà di parola che spuntava là fuori” (Celati, I987, p. 88) - un'apparenza che le sue parole cercano nondimeno di rendere misteriosamente presente. Le tante conclusioni inconclusive di Tabucchi, le attività non 
rivelate ai lettori di molti dei suoi personaggi suggeriscono che anche nel suo mondo immaginario ci siano certe presenze che lui, come Pereira davanti al suo inquisitore, preferirebbe non indicare direttamente. Anche Bufalino è sensibile ai limiti delle sue creazioni artistiche, limiti che lo fanno soffrire, come dice egli stesso con un'espressione caratteristicamente forte, dal "dubbio, scrivendo, di castrarsi irrimediabilmente, costringendosi a comprimere fra due costole di libro un magma infinito" (Bufalino, p. 6). Questo magma, vorrei suggerire in conclusione, non è altro che l'assenza/presenza, mancanza/infinito descritta da scrittori come Natoli, Vattimo, Gentiloni ed Eco, che costituisce il senso percepibile anche se non descrivibile "del religioso" (cfr. Parmeggiani, passim).

\section{Bibliografia}

Acunti G.D., "Alla ricerca della sacralità della parola. Vincenzo Consolo", in Parola di scrittore, a cura di V. Della Valle, Roma, Minimum fax, I997, pp. IOI-II6.

Bufalino G., Conversazione con Gesualdo Bufalino. Essere o riessere, a cura di P. Gaglianone e L. Tas, Nota critica di N. Zago, Roma, Omicron, 1996.

Calvino I., Usi politici giusti e sbagliati della letteratura, in Una pietra sopra. Discorsi di letteratura e società, Torino, Einaudi, 1980, pp. 286-293.

Capati M., Storia letteraria del 'goo italiano, Venezia, Marsilio, 2002.

Celati G., Meteorite dal cosmo, in Narratori delle pianure, Milano, Feltrinelli, I985, pp. 7I-76.

-, I lettori di libri sono sempre piu falsi, in Quattro novelle sulle apparenze, Milano, Feltrinelli, Milano, 1987, pp. 63-95.

-, Avventure in Africa, Milano, Feltrinelli, 1998.

-, Vite di pascolanti. Tre racconti, Roma, Nottetempo, 2006.

Croce B., Perché non possiamo non dirci cristiani, in Discorsi di varia filosofia, vol. I, Bari, Laterza, 1945, pp. II-23.

Eco U. e Martini C.M., In cosa crede chi non crede?, Roma, Libera Atlantide, 1996.

Gentiloni F., Abramo contro Ulisse. Un itinerario alla ricerca di Dio, Torino, Claudiana, 2003.

Gumpert C., "La letteratura come enigma ed inquietudine. Una conversazione con Antonio Tabucchi", in Dedica a Antonio Tabucchi, a cura di C. Cattaruzza, Pordenone, Associazione Provinciale per la Prosa, 200I, pp. I7-IO5. 
Natoli S., Dio e il divino. Confronto con il cristianesimo, Brescia, Morcelliana, I999.

-, Il cristianesimo di un non credente, Magnano, Qiqajon, 2002.

-, Parole della filosofia o dell'arte di meditare, Milano, Feltrinelli, 2004. Parmeggiani F., Lo spessore della letteratura. Presenze neotestamentarie nella narrativa italiana degli anni Sessanta e Settanta, Ravenna, Longo, 2007.

Ragone G., Un secolo di libri. Storia dell'editoria in Italia dall'Unità al post-moderno, Torino, Einaudi, 1999.

Spunta M., Voicing the Word: Orality in Contemporary Italian Fiction, Bern, Peter Lang, 2004.

Vаттімо G., Credere di credere, Milano, Garzanti, 1996.

—, Dopo la cristianità: per un cristianesimo non religioso, Milano, Garzanti, 2002.

Vattimo G., Derrida J. e Gadamer H.-G., La Religion : Séminaire de Capri, Paris-Bari, Seuil-Laterza, 1966.

Vattimo G. e Paterlini P., Non essere Dio, Reggio Emilia, Aliberti, 2006.

Vattimo G., Sequeri P. e Ruggeri G., Interrogazioni sul cristianesimo. Cosa possiamo ancora attenderci dal Vangelo?, Roma, Edizioni Lavoro, 2000. 
